\title{
What is a COPD-like spirometry test result in resource constrained settings?
}

Gonzalo E Gianella ${ }^{\mathrm{a}, \mathrm{b}}$, J. Jaime Miranda ${ }^{\mathrm{a}, \mathrm{b}}$, John R Hurst ${ }^{\mathrm{c}}$

a. Department of Medicine, School of Medicine, Universidad Peruana Cayetano Heredia, Lima, Peru

b. CRONICAS Center of Excellence in Chronic Diseases, Universidad Peruana Cayetano Heredia, Lima, Peru

c. UCL Respiratory, University College London, London, UK

\section{Correspondence to:}

Dr Gonzalo Gianella, MD

CRONICAS Centre of Excellence in Chronic Diseases

Universidad Peruana Cayetano Heredia

Address: Av. Armendáriz 445, Miraflores, Lima 18, Peru

E-mail: gonzalo.gianella.m@ upch.pe 


\begin{abstract}
The diagnosis of chronic obstructive pulmonary disease (COPD), a global health problem, is challenging in resource-constrained settings. Spirometry with an obstructive pattern after the administration of bronchodilators is required for the diagnosis of COPD. Existing COPD treatment guidelines, largely derived from studies performed in populations of cigarette-smokers, recommend pharmacologic interventions with a tendency to include new - and expensivedrugs as first line agents. As the different factors that cause non-smokers to develop COPD lead to different phenotypes of disease, COPD severity and treatment efficacy cannot be extrapolated to be the same as in the population of smokers. In so doing, current global initiatives may carry risks when trying to over simplify diagnostic approaches and push for standardization of treatment algorithms that are not context-specific. Future work to mitigate the global burden of COPD needs to address the need for new epidemiological data, especially in areas where tobacco use is less prevalent and environmental factors such as domestic air pollution are common.
\end{abstract}




\section{What is a COPD-like spirometry test result in resource constrained settings?}

Chronic Obstructive Pulmonary Disease (COPD) is a global health problem [1] that poses a major burden to people living with the condition, through severity-related respiratory symptoms, function impairment and exacerbations, and the development of comorbidities and complications. But there is one major caveat: COPD has been mainly studied in high income setting and largely in cigarette smokers.

Although tobacco is the best-known cause for COPD, nonsmokers also develop chronic respiratory health problems. Nonsmokers' COPD has been attributed to a large and diverse list of factors that range from exposure to environmental/occupational fumes, impaired lung development and consequences of chronic infections such as tuberculosis. From a global health perspective, or as more recently suggested from a precision public health angle, transposing COPD management recommendations to other settings requires, as a minimum, some scrutiny.

Tobacco produces a well-studied cascade of patho-physiological consequences that affect both the airway and lung parenchyma [2]: chronic airway inflammation/damage (chronic bronchitis), small airway narrowing and lung parenchymal destruction (emphysema). Although airway diseases can be seen with exposure to other inhaled fumes, significant emphysema in clinical practice is almost exclusively due to tobacco and other drugs of addiction.

Emphysema, small airway obstruction and chronic bronchitis can cause obstruction on spirometry. Whereas chronic bronchitis causes mainly an airway problem, in emphysema small airways inflammation secondary to tobacco mediates the destruction of the normal lung architecture. As normal exhalation depends on the lung's elastic properties (highly dependent on surrounding alveolar walls), the parenchymal destruction seen in emphysema enhances the dynamic compression of the small airways, generating the typical expiratory flow limitation seen in tobacco related COPD.

Spirometry with an obstructive pattern after the administration of bronchodilators (fixed airway obstruction) is required for the diagnosis of COPD [1]. As most chronic respiratory conditions share similar symptoms (cough, dyspnea and phlegm) spirometry results tend to direct COPD diagnosis, an oversimplification that may lead to misdiagnosis. As an example, conditions such as under-treated asthma and bronchiectasis can also be associated with fixed airway obstruction. Also, treated asthmatics do not immediately normalize spirometry after bronchodilators to a nonobstructive pattern.

To define airway obstruction, current guidelines favor the use of a fixed FEV1/FVC ratio [1], yet it has been shown the prevalence of COPD increases significantly when the recommended fixed ratio of 0.70 is used, resulting in overdiagnosis of COPD [3]. In populations with lower pre-test probability, as in older non-smokers, the risk of overdiagnosis appears to be more significant $[3,4]$. Defining airway obstruction using the lower limit of normality has been recommended as an alternative to improve the positive predictive value of spirometry [5]. However, the absence of reference values for different populations, from where the lower limits of normality values can be derived, is an important obstacle in limited resource settings.

As access to treatment depends on several factors, fixed airway obstruction in a setting with a responsive health system (one that guarantees access to services and pharmacological treatment) and high tobacco consumption will correlate well with smoking-related COPD. Yet, the same clinical scenario in a reality with a deficient health system and low tobacco consumption may well be the reflection of other diseases, including cases of never or under-treated asthma or structural lung disease secondary to tuberculosis. 
Tobacco-related COPD is often accompanied by frequent exacerbations and hospitalizations, especially if significant lung parenchymal destruction has been established. In emphysema, hypoxemia due to alveolar destruction and ventilation/perfusion mismatch is associated with increased mortality. Although the knowledge of the natural history of COPD in non-smokers is limited, studies in this group of patients typically show absence of emphysema, less functional impairment and slower decline in lung function $[6,7]$.

COPD treatment guidelines recommend pharmacologic interventions that are based in studies performed in smokers, with a tendency to include new (and expensive) drugs as first line agents. Inhaled steroids (ICS) are not recommended as first-line agents for stable COPD. Clinical studies show that in tobacco-related COPD ICS use may increase the risk of pneumonia with a comparable efficacy to non-steroid containing inhalers [8,9]. Also, there is increasing evidence that ICS use in COPD should be guided by biomarkers such as blood eosinophils [10]. On the other hand, ICS remain central in asthma treatment.

There are risks when global initiatives try to over simplify diagnosis approaches and push for standardization of treatment algorithms. As the different factors that cause COPD in non-smokers may lead to different phenotypes of disease, severity cannot be extrapolated. If low quality health systems are added to the equation, the risk of misdiagnosis of alternative, untreated respiratory conditions also increases. Add to this complexity the heterogeneity in disease burden, e.g. high prevalence of tuberculosis compounded with low prevalence of smoking, the rationale for a onesize-fits-all approach becomes challenged and even potentially inappropriate.

Future work to mitigate the global burden of COPD needs to address the need for new epidemiological data, especially in areas where tobacco use is less prevalent and environmental factors such as domestic air pollution are common. In addition to better characterization of different phenotypes of the disease, enrolment of non-smokers in clinical trials remains a big challenge. Until then, due to the absence of robust data, caution is advised in generalizing recommendations about COPD, particularly in low and middle-income settings.

\section{Conflict of interests}

The authors declare no conflicts of interest.

\section{References}

1. Global Initiative for Chronic Obstructive Lung Disease. Global strategy for the diagnosis, management, and prevention of chronic obstructive pulmonary disease. Fontana: Gold; 2019 [cited 2019 May 23]. Available from: https://goldcopd.org/wp-content/uploads/2018/11/GOLD-2019-v1.7-FINAL14Nov2018-WMS.pdf

2. Barnes PJ. Cellular and molecular mechanisms of chronic obstructive pulmonary disease. Clin Chest Med. 2014;35(1):71-86.

3. Hansen, James E. et al. Spirometric Criteria for Airway Obstruction. CHEST, Volume 131, Issue 2, 349 - 355

4. Astri Medbø, Hasse Melbye. Lung function testing in the elderly — Can we still use FEV1/FVCo70\% as a criterion of COPD? Respiratory Medicine (2007) 101, 10971105

5. Swanney MP, Ruppel G, Enright PL, et al. Using the lower limit of normal for the $\mathrm{FEV}_{1} / \mathrm{FVC}$ ratio reduces the misclassification of airway obstruction. Thorax 2008;63:1046-1051.

6. Ramirez-Venegas A, Sansores RH, Quintana-Carrillo RH, et al. FEV1 decline in patients with chronic obstructive pulmonary disease associated with biomass exposure. Am J Respir Crit Care Med. 2014;190(9):996-1002. 
7. Torres-Duque CA, Garcia-Rodriguez MC, Gonzalez-Garcia M. Is Chronic Obstructive Pulmonary Disease Caused by Wood Smoke a Different Phenotype or a Different Entity? Arch Bronconeumol. 2016;52(8):425-31.

8. Kew KM, Seniukovich A. Inhaled steroids and risk of pneumonia for chronic obstructive pulmonary disease. Cochrane Database Syst Rev. 2014;10(3):CD010115.

9. Wedzicha JA, Banerji D, Chapman KR, et al. Indacaterol-Glycopyrronium versus Salmeterol-Fluticasone for COPD. N Engl J Med. 2016;374(23):2222-34.

10. Bafadhel M, Peterson S, De Blas MA, et al. Predictors of exacerbation risk and response to budesonide in patients with chronic obstructive pulmonary disease: a posthoc analysis of three randomised trials. Lancet Respir Med. 2018;6(2):117-126. 